\title{
Application of Semantic Feature Analysis as a Treatment for Aphasic Dysnomia
}

\author{
Mary Boyle \\ The Burke Rehabilitation Hospital, White Plains, NY \\ Carl A. Coelho \\ Southern Connecticut State University, New Haven, CT
}

Despite agreement that dysnomia affects virtually every aphasic patient, there is no consensus about the purpose and effectiveness of techniques to treat it. Semantic feature analysis (SFA), a treatment technique designed to improve retrieval of conceptual information by accessing semantic networks, was used to treat aphasic dysnomia in a 57-year-old male who exhibited Broca's aphasia secondary to a left frontoparietal ischemic infarction. SFA was effective for improving confrontation naming and for generalized improvement to untreated pictures. However, no generalization to connected speech was seen on the measures of mean words per minute, mean correct information units per minute, or the percentage of all words that were correct information units.

D espite agreement that dysnomia affects virtually every aphasic patient, there is no consensus about the purpose and effectiveness of techniques to treat it. For example, Brookshire (1992) questions the pragmatic value of confrontation naming exercises, whereas Shewan and Bandur (1986) consider them to be a means of facilitating general lexical retrieval processes. A review of the dysnomia treatment literature reveals: (a) conflicting results regarding generalization to untreated items (Howard, Patterson, Franklin, Orchard-Lisle, \& Morton, 1985a, 1985b; Thompson \& Kearns, 1981; Thompson, Raymer, \& le Grand, 1991); (b) insufficient description of techniques to allow replication (Seron, Deloche, Bastard, Chassin, \& Hermand, 1979; Wiegel-Crump \& Koenigsknecht, 1973); (c) lack of experimental control (Howard et al., 1985a, 1985b; Marshall, Pound, White-Thomson, \& Pring, 1990); (d) no assessment of maintenance ( $\mathrm{Li} \&$ Canter, 1987, 1990; Linebaugh \& Lehner, 1979); and (e) no assessment of generali- zation to speaking situations other than confrontation naming (Howard et al., 1985a, 1985b; Marshall et al., 1990; Seron et al., 1979; Thompson \& Kearns, 1981; Thompson et al., 1991; WiegelCrump \& Koenigsknecht, 1973).

This study was designed to assess the efficacy of semantic feature analysis (SFA) (Ylvisaker \& Szekeres, 1985) as a treatment for aphasic dysnomia, in terms of generalization to untreated items, generalization to connected speech, and change in the perception of a significant other. It was also designed to assess the efficacy of training sufficient exemplars as a strategy to enhance generalization (Rosenbek, LaPointe, \& Wertz, 1989; Stokes \& Baer, 1977; Thompson, 1989) which, according to Thompson (1989, p. 100 ), has not been specifically addressed in studies of aphasia.

Semantic feature analysis is thought to improve retrieval of conceptual information by accessing semantic networks (Massaro \& Tompkins, 1992; Ylvisaker \& Szekeres, 1985). During SFA treatment, the patient is encouraged to produce words semantically related to the target. According to the spreading activation theory of semantic processing (Collins \& Loftus, 1975), by activating the semantic network surrounding the target, the target itself should be activated above its "threshold" level, thus increasing the likelihood that its name can be retrieved. Some success with this technique has been reported for two subjects with traumatic brain injury (Massaro \& Tompkins, 1992), but there are no reports on its use with aphasic adults.

The research questions addressed in this study were: (a) What are the effects of SFA treatment on confrontation naming of trained and untrained exemplars in an adult with aphasic dysnomia, on measures of communicative informa- tiveness and efficiency, and on the perception of a significant other regarding the subject's communicative effectiveness? and (b) Does using a larger number of stimuli promote generalization more efficiently than using the same small number of stimuli in each session?

\section{Method}

\section{Subject}

The subject, HW, was a 57-year-old right-handed native-English-speaking male who was 65 months postonset of a left frontoparietal ischemic infarction. He was a widowed retired postal worker with a high school education. HW passed a pure-tone hearing screening at $30 \mathrm{~dB}$ HL. Administration of the Western Aphasia Battery (WAB; Kertesz, 1982) revealed a Broca's aphasia with a mild apraxic component. HW's WAB Aphasia Quotient was 82 . Examination of the oral articulators revealed normal strength, range of motion, and coordination during nonspeech movements.

Dysnomia was a prominent characteristic of HW's spontaneous speech, as evidenced by frequent long pauses during which he seemed to be searching for a word. Sometimes he produced nonword fillers (e.g., "uh" or "um") or incomplete word attempts during these pauses. When questioned during such pauses, HW indicated that he couldn't think of the word he wanted to say. Sometimes he was able to produce the desired word at the end of the pause, and sometimes not. Dysnomia was also evident on formal testing. HW's score on the Boston Naming Test (Goodglass \& Kaplan, 1983) was 43 out of 60 , and his score on the Test of Adolescent/Adult Word Finding (German, 1990) was 67 out of 107. HW did not receive any other 
speech-language treatment during the study.

\section{Stimuli}

The 260 black and white line drawings developed and standardized for name agreement, image agreement, familiarity, and visual complexity by Snodgrass and line drawings were enlarged to approximately $4 \mathrm{~cm}$ by $6.5 \mathrm{~cm}$ and individually mounted on $7.5 \mathrm{~cm}$ by $13 \mathrm{~cm}$ index cards. These pictures were presented to HW for naming across three trials without any cueing or feedback. Results of these baseline measures can be seen in Figure 1. The seven pictures that HW was unable to name during any trial were chosen as control pictures. There were 34 pictures that he could not name on two of the three trials, and these were chosen as treatment pictures and divided as follows: seven were randomly chosen for use during the Few Exemplars condition and the remaining 27 were assigned to the Many Exemplars condition. In addition, seven pictures that $\mathrm{HW}$ was able to name during all three trials (easy pictures) were chosen to provide periodic success during treatment sessions; responses to these were not used as data.

To assess HW's recognition of the 41 pictures that were chosen as control and treatment stimuli, a comprehension task was administered. A picture plate was prepared for each of the 41 items, consisting of the target picture and five foils. HW was asked to point to a target on each picture plate after it was named aloud. He made no errors on this task, suggesting that his inability to name the Vanderwart (1980) served as stimuli. The

41 pictures was not because he did not recognize the pictures.

\section{Design}

An A-B single-subject design was used. Baseline, probe, and maintenance measures of the control pictures and of the informativeness and efficiency of HW's connected speech were collected to assess the effectiveness, generalization, and maintenance of treatment. The connected speech elicitation stimuli were the five tasks designated as Set A by Nicholas \& Brookshire (1993). Set A consists of two black and white line drawings of complex scenes, one black and white line-drawn picture sequence that relates a story, one request for personal information ("Tell me what you usually do on Sundays"), and one request for procedural information ("Tell me how you would go about doing dishes by hand"). Pretreatment and posttreatment social validity measures were also collected. HW's daughter, a 20-yearold college student who lives with him, served as the rater of his communication ability for the social validity measures.

\section{Treatment}

HW attended three 60 -minute treatment sessions per week. Two treatment conditions were used. In the Few Exemplars condition, the same seven pictures were used repeatedly in each session. The seven easy pictures were interspersed with the treatment pictures to provide periodic success. The treatment and easy pictures were shuffled before each session to prevent any order effect. Each picture was presented only once per session.

FIGURE 1. The percentage of the 260 Snodgrass and Vanderwart (1980) pictures named correctly by $\mathrm{HW}$ across three baseline sessions.

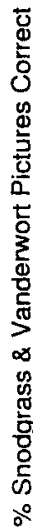

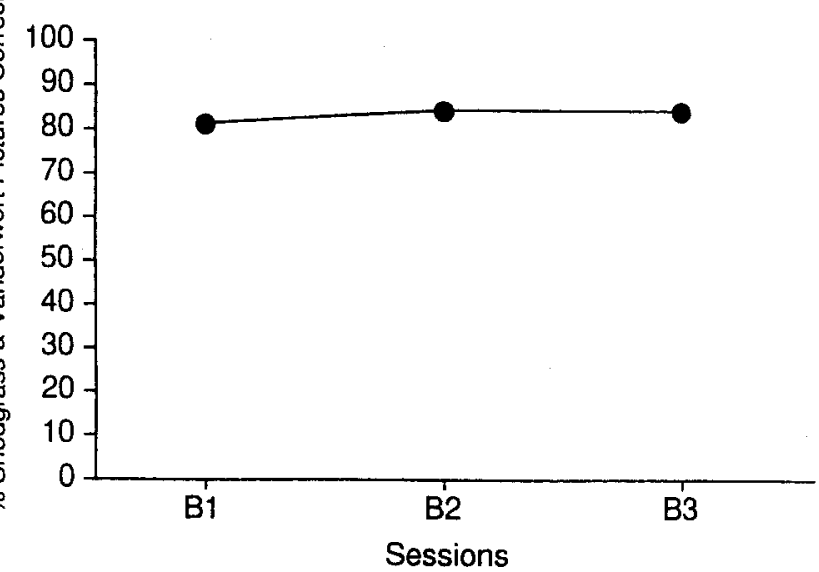

In the Many Exemplars condition. the remaining 27 pictures were randomized into three sets of nine to be used in successive treatment sessions that week. The seven easy pictures were interspersed into each set before treatment sessions. Each picture was presented only once per session.

Following three baseline sessions, HW was entered into the Few Exemplars condition until he achieved $100 \%$ accuracy on the treatment pictures for three consecutive sessions. Following a oneweek break, he was entered into the Many Exemplars condition until he achieved $100 \%$ accuracy for three consecutive sessions. Probes were administered at the end of the last treatment session each week. The probes consisted of confrontation naming of the control pictures and responses to the connected speech elicitation stimuli. No cues or specific feedback were provided during probe tasks.

Semantic feature analysis was used in both treatment conditions. A picture was placed in the center of the feature analysis chart (Figure 2) and HW was asked to name the picture. Next, regardless of his ability to name the picture, HW was guided in verbalizing the semantic features of each target with the aid of the chart and cues from the clinician. The clinician wrote the semantic features in the appropriate locations on the chart after $\mathrm{HW}$ said them. If HW couldn't provide a feature, the clinician provided it orally and in writing. Sometimes he was able to name the target after one or more of the features were listed. In order to establish and encourage use of the technique, all features were produced for all pictures, even those that HW had named on initial confrontation or those that he named during semantic feature production. If $\mathrm{HW}$ was still unable to name the picture after all features were written on the chart, the clinician said the name aloud and required HW to repeat it and to review all its features.

\section{Data Preparation and Analysis}

Responses to all baseline and probe tasks were audio recorded and transcribed verbatim. The printed transcriptions of the narratives were used for scoring words and correct information units (Nicholas \& Brookshire, 1993). Transcription and scoring were done by the first author. To assess reliability, the second author independently scored $20 \%$ of the speech samples collected during baseline and probe sessions, and the first author rescored $10 \%$ of the samples 5 weeks after they had been scored initially. Point-to-point inter- and intrarater agreement exceeded $99 \%$ for accuracy of transcription and exceeded $95 \%$ 
FIGURE 2. The feature analysis chart used during treatment sessions. HW was guided in producing the semantic features of each target, and the features were written in the appropriate locations on the chart.

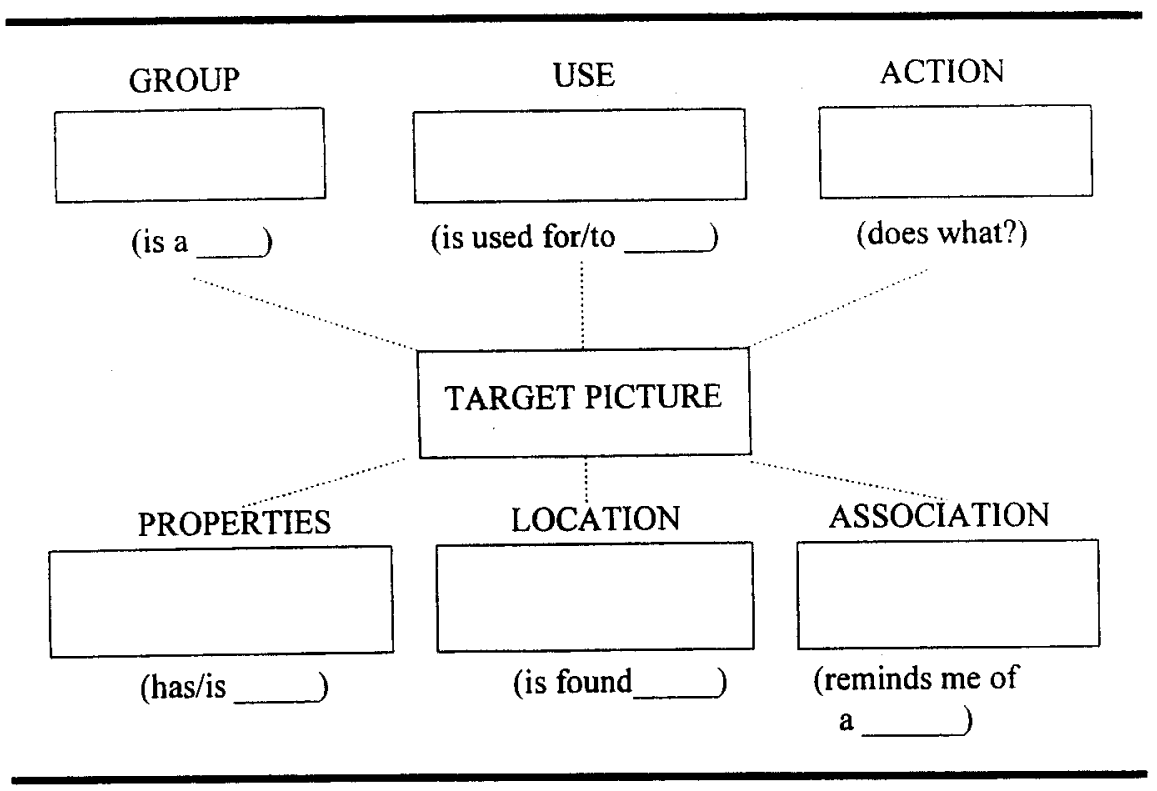

FIGURE 3. The percentage of treatment and control pictures named correctly by HW during baseline sessions, the two treatment conditions, probe sessions, and maintenance (FU) sessions.

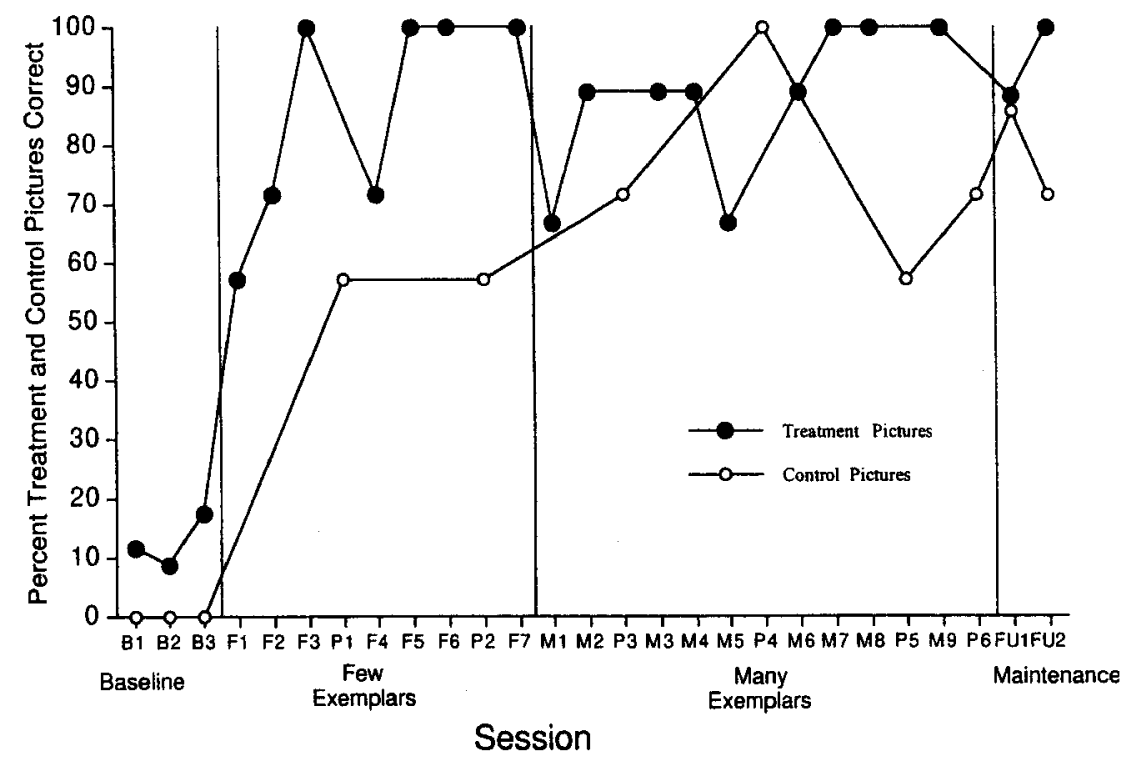

for all dependent measures. Discrepancies in transcription and scoring were resolved through discussion.

\section{Results}

\section{Effectiveness of Treatment}

HW named fewer than $20 \%$ of the treatment pictures correctly during baseline sessions (see Figure 3). His performance improved in the Few Exemplars condition until he achieved criterion during the seventh session. Performance declined when the Many Exemplars condition was initiated following a week-long break, then improved until criterion was achieved in session M9. Follow-up indicated that the effect of treatment was maintained for 1 and 2 months post-treatment.
Generalization of Confrontation Naming to Untreated Pictures

Performance on the control pictures improved during the Few Exemplars condition and remained at approximately the same level during the Many Exemplars condition (see Figure 3). The generalization effect was maintained 1 and 2 months after treatment had been discontinued.

\section{Generalization to Connected Speech}

To assess generalization of improved confrontation naming to connected speech, Nicholas and Brookshire's (1993) procedures for calculating mean words per minute, mean correct information units (CIUs) per minute, and the percentage of all words that were CIUs were used. Figures 4 and 5 show that no changes from baseline levels occurred on these measures during or after treatment.

\section{Social Validity of Changes}

The Communicative Effectiveness Index (Lomas et al., 1989) was completed by the patient's daughter before treatment and during the final probe session. HW received a pretreatment score of 65.56 and a posttreatment score of 77.56 , yielding a change score of 12.00. According to Lomas and his colleagues (1989), this represents a clinically important improvement.

\section{Discussion}

Semantic feature analysis was associated with improved confrontation naming on trained and untrained items, but not with improved connected speech measures. Generalization occurred when only a small number of exemplars were trained. The treatment and generalization effects were maintained for 2 months after treatment was discontinued.

There are two studies with welldefined treatment methodologies that reported generalization of improved naming to untreated items. Hillis (1989) used a single-subject design replicated across two subjects with aphasia to investigate the effects of a cueing hierarchy on written naming of black and white line drawings. The cueing hierarchy was associated with generalization for only one subject. It consisted of two successive scrambled anagrams of the name, the initial letter of the name, the verbal name, and the written name.

There are obvious differences between semantic feature analysis and the treatment used by Hillis. Semantic feature analysis required the subject to provide 
semantic information about the target. The experimenter provided information only when the subject could not. In contrast, Hillis' hierarchy relied primarily on the experimenter's provision of cues, which were primarily graphemic or phonologic. Since both Hillis' treatment and semantic feature analysis resulted in generalization to untrained items, there may be several methods that can result in improved confrontation naming. A direct comparison of these treatments is necessary to determine whether one is more effective and/or efficient than the other. Hillis did not provide data on maintenance effects of her treatment, so it cannot be compared to SFA in this regard. It remains to be seen whether her treatment remains effective beyond the treatment period.

Howard and his colleagues (1985b) investigated the effects of semantic and phonologic treatments on naming black and white line drawings by 12 adults with aphasia. The semantic treatment required subjects to point to one of four pictures on command, to match a written word to one of four pictures, and to answer a yes/no question requiring a semantic judgment about the item (e.g., "Is a cat an animal?"). The phonologic treatment required subjects to repeat the item's name, produce the name after a phonemic cue, and to make a rhyming judgment about the name. Howard and his colleagues reported that both treatments resulted in improved naming, but that improvements made during treatment were not maintained 6 weeks after therapy ended.

These results suggest that confrontation naming might be improved via either a semantic or a phonologic route. The lack of maintenance effects is discouraging, however. Howard and colleague's treatments relied primarily on cues that were provided by the experimenter. Perhaps Semantic Feature Analysis had a more lasting effect because SFA requires the subject, rather than the experimenter, to generate information about the target. Although the feature analysis chart contains prompts for this information, the prompts in the chart might be internalized after several repetitions of its use, allowing the subject to self-cue when the chart is not present. In contrast, it is unlikely that a subject could self-cue using Howard and colleague's techniques, since all of the cues except the initial phoneme cue require the target's name. Obviously, this is highly speculative. Nevertheless, direct comparison of treatments that lend themselves to self-cueing and those that require clinician cueing might yield important information regarding maintenance of improvements in confrontation naming.

No generalization to connected speech

FIGURE 4. The mean words per minute and correct information units (CIUs) per minute produced by $\mathrm{HW}$ in response to the connected speech stimuli during baseline, probe, and follow-up sessions.

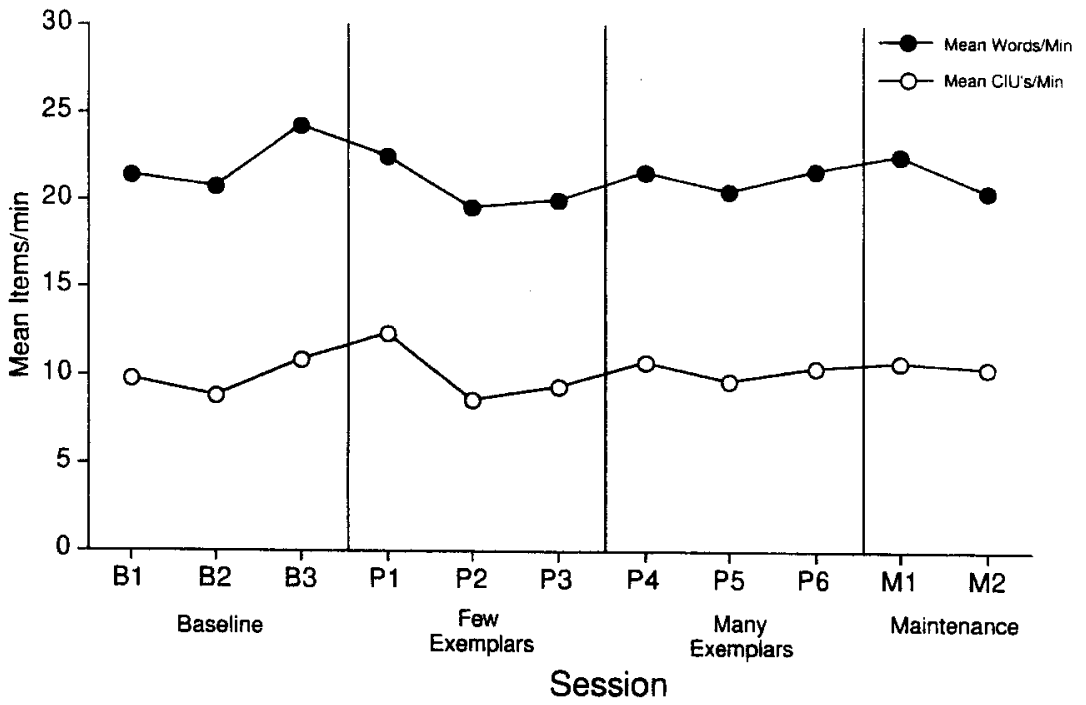

FIGURE 5. The percentage of all words that were correct information units (CIUs) produced by $\mathrm{HW}$ in response to the connected speech stimuli during baseline, probe, and follow-up sessions.

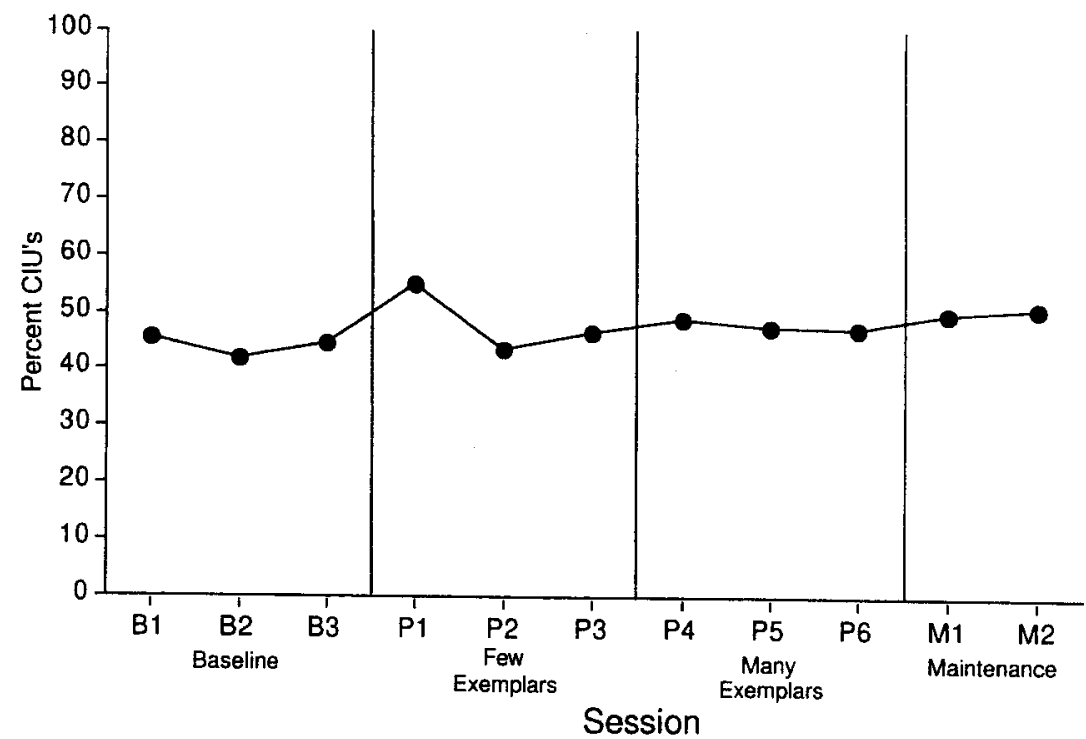

was seen on the measures used in this study. This result suggests that direct treatment of word retrieval in connected speech might be necessary to achieve an improvement at that level of discourse.

Despite the lack of improvement on the connected speech measures, HW's daughter judged his speech to have improved.
Perhaps this was simply a result of her desire for her father's speech to improve and/or for his treatment to continue. Alternatively, it could be that changes did occur in his connected speech, but the measures of informativeness and efficiency used in this study were not sensitive to these changes. 
Finally, generalization of improved confrontation naming to untreated pictures occurred when as few as seven exemplars were treated. At least for $\mathrm{HW}$, seven exemplars was a sufficient number to achieve generalization, and scores on the untreated pictures did not improve significantly when additional exemplars were trained. Whether such a small number of exemplars is sufficient for other subjects with aphasia is not clear and requires replication of this study with other subjects. Such replication is necessary, in fact, before any of these results can be generalized to the aphasic population.

\section{References}

Brookshire, R. H. (1992). An introduction to neurogenic communication disorders. St. Louis, MO: Mosby-Year Book, Inc.

Collins, A. M., \& Loftus, E. F. (1975). A spreading activation theory of semantic processing. Psychological Review, 52, $407-428$.

German, D. J. (1990). National College of Education Test of Adolescent/Adult Word Finding. Allen, TX: DLM.

Goodglass, H., \& Kaplan, E. (1983). Boston Naming Test. Philadelphia: Lea \& Febiger.

Hillis, A. E. (1989). Efficacy and generalization of treatment for aphasic naming errors. Archives of Physical Medicine and Rehabilitation, 70, 632-636.

Howard, D., Patterson, K. J., Franklin, S., Orchard-Lisle, V., \& Morton, J. (1985a). The facilitation of picture naming in aphasia. Cognitive Neuropsychology, 2, 163-190.

Howard, D., Patterson, K. J., Franklin, S., Orchard-Lisle, V., \& Morton, J. (1985b). Treatment of word retrieval deficits in aphasia: A comparison of two therapy methods. Brain, 108, 817-829.

Kertesz, A. (1982). Western Aphasia Battery.
New York: The Psychological Corporation.

Li, E. C., \& Canter, G. J. (1987). An investigation of Luria's hypothesis on prompting in aphasic naming disturbances. Journal of Communication Disorders, 20, 469-475.

Li, E. C., \& Canter, G. J. (1990). The effects of grammatic class and cue type on cueing responsiveness in aphasia. Brain and Language, 38, 48-60.

Linebaugh, C., \& Lehner, L. (1979). Cueing hierarchies and word retrieval: A therapy program. In R. H. Brookshire (Ed.), Clinical aphasiology: 1979 (Vol. 9, pp. 19-31). Minneapolis: BRK Publishers.

Lomas, J., Pickard, L., Bester, S., Elbard, H., Finlayson, A., \& Zoghaib, C. (1989). The Communicative Effectiveness Index: Development and psychometric evaluation of a functional communication measure for adult aphasia. Journal of Speech and Hearing Disorders, 54, 113-124.

Marshall, J., Pound, C., White-Thomson, M., \& Pring, T. (1990). The use of picture/ word matching tasks to assist word retrieval in aphasic patients. Aphasiology, 4, 167184.

Massaro, M. E., \& Tompkins, C. A. (1992). Feature analysis for treatment of communication disorders in traumatically braininjured patients: An efficacy study. Clinical Aphasiology, 22, 245-256.

Nicholas, L. E., \& Brookshire, R. H. (1993). A system for quantifying the informativeness and efficiency of the connected speech of adults with aphasia. Journal of Speech and Hearing Research, 36, 338-350.

Rosenbek, J. C., LaPointe, L. L., \& Wertz, R. T. (1989). Aphasia: A clinical approach. Boston: College-Hill.

Seron, X., Deloche, G., Bastard, V., Chassin, G., \& Hermand, N. (1979). Word-finding difficulties and learning transfer in aphasic patients. Cortex, 15, 149-155.

Shewan, C. M., \& Bandur, D. L. (1986). Treatment of aphasia: A language-oriented approach. San Diego: College-Hill.

Snodgrass, J. G., \& Vanderwart, M. (1980).
A standardized set of 260 pictures: Norms for name agreement, image agreement, familiarity, and complexity. Journal of Experimental Psychology: Human Leaming and Memory, 6, 174-215.

Stokes, T., \& Baer, D. (1977). An implicit technology of generalization. Journal of Applied Behavior Analysis, 10, 349-367.

Thompson, C. (1989). Generalization in the treatment of aphasia. In L. V. McReynolds \& J. E. Spradlin (Eds.). Generalization strategies in the treatment of communication disorders (pp. 82-115). St. Louis, MO: B. C. Decker.

Thompson, C., \& Kearns, K. (1981). Experimental analysis of acquisition, generalization, and naming behaviors in a patient with anomia. In R. H. Brookshire (Ed.), Clinical Aphasiology: 1981 (Vol. 11, pp. 35-45). Minneapolis: BRK Publishers.

Thompson, C. K., Raymer, A., \& le Grand, H. (1991), Effects of phonologically based treatment on aphasic naming deficits: A model-driven approach. Clinical Aphasiology, 20, 239-261.

Wiegel-Crump, C., \& Koenigsknecht, R. A. (1973). Tapping the lexical store of the adult aphasic: Analysis of the improvement made in word retrieval skills. Cortex, 9 , 411-418.

Ylvisaker, M., \& Szekeres, S. (1985, Novem ber). Cognitive-language intervention with brain-injured adolescents and adults. Miniseminar presented at the annual convention of the Illinois Speech-Language-Hearing Association.

Contact author: Mary Boyle, PhD, Burke Rehabilitation Hospital, 785 Mamaroneck Avenue, White Plains, NY 10605

Key Words: aphasia, aphasia treatment, dysnomia (or anomia), semantic feature analysis, language disorders 